\title{
ASPECTOS BIOLOGICOS DE Panacea prola amazonica (FRUHSTORFER) (LEPIDOPTERA: NYMPHALIDAE), EN LA AMAZONIA PERUANA
}

\author{
Joel VÁSQUEZ ${ }^{1}$, Gerardo LAMAS², Guy COUTURIER ${ }^{3}$, Kember MEJIA $^{1}$ \\ 1 Instituto de Investigaciones de la Amazonía Peruana - IIAP, Programa de Investigación en Biodiversidad Amazónica - \\ PIBA, Apartado 784, Iquitos, Perú. E-mail: jvasquez@iiap.org.pe \\ 2 Universidad nacional Mayor de San Marcos, Museo Nacional de Historia Natural, Avenida Arenales, Jesús María, Lima, \\ Perú. E-mail : gerardo@musm.edu.pe \\ 3 Muséum National d'Histoire Naturelle, Département Systématique et Evolution, case 50, 57 rue Cuvier, 75231 Paris \\ FranciaE-mail couturie@mnhn.fr
}

\begin{abstract}
RESUMEN
Los resultados del estudio muestran que Panacea prola amazonica copula entre el tercer y séptimo día de la emergencia. La oviposición, se realiza en hojas y ramas, está relacionada con la aparición de brotes de la planta hospedera "metohuayo" Caryodendron orinocense Karst. Su capacidad promedio de oviposición es de $262.80 \pm 119.0$ huevos. La duración del ciclo, desde huevo a adulto fue de 26 a 37 días, con 6 estadíos larvales. Los adultos nacen entre las 8.00 a $11.00 \mathrm{am}$, los machos sobreviven de 6 a 9 y las hembras aproximadamente 30 días en cautiverio. Se encontraron siete enemigos naturales, un parasitoide de huevo, tres parasitoides de larvas, un parasitoide de pre pupa, un depredador de larva, un depredador de adulto y un hongo entomopátogeno de pupa.
\end{abstract}

PALABRAS CLAVE: Manejo de mariposas, Panacea prola amazónica, Amazonía peruana, Caryodendron orinocense

\section{THE BIOLOGICAL ASPECTS OF PANACEA PROLA AMAZONICA (FRUHSTORFER) (LEPIDOPTERA: NYMPHALIDAE), IN PERUVIAN AMAZON}

\begin{abstract}
The results show that the copulation of the Panacea prola occurs between the third and seventh day of the hatching. The oviposition, occurs in leaves and branches, is related to outbreaks of the host plant "metohuayo" Caryodendron orinocense Karst. The average capacity of oviposition is $262.80 \pm 119.0$ eggs. The cycle from egg to adult takes 26 to 37 days, with six larval phases. Adults are born from 8.00 to $11.00 \mathrm{am}$, the males survive between 6 a 9 days and females about 30 days in captivity. We found seven natural enemies, an egg parasitoid, three larvae parasitoids, a pre pupal parasitoid, a larvae predator, an adult predator and a pupal entomopathogenic fungus.
\end{abstract}

KEYWORDS: Butterfly management, Panacea prola amazonica, Peruvian Amazon, Caryodendron orinocense 


\section{INTRODUCCIÓN}

Panacea prola amazonica (Fruhstorfer, 1915) es una mariposa tropical distribuida en la Amazonia del Ecuador y Perú (Robbins et al., 1996; Hill et al., 2002; Checa et al., 2009), y observaciones reciente por Salazar et al., 2010 indican su presencia en Colombia. La única planta hospedera encontrada para Panacea prola amazónica en la amazonia del Perú ha sido Caryodendron orinocense Karst. (Figura 1). Es una planta autóctona y endémica de la Orinoquia y la Amazonia (Martínez, 1970). Es un árbol mediano a grande que puede alcanzar más de $20 \mathrm{~m}$ de altura y de corteza moteada (Gonzales \& Torres, 2010). No existe ningún reporte de los aspectos biológicos de esta sub especies de mariposa Montoya (1989) reporta los aspectos biológicos de Panacea sp., posible prola, denominado "gusano cachon del inchi" cuyas larvas fueron encontradas en Caryodendron orinocense H. Karts (Euphorbiaceae). Observaciones recientes indican que las larvas de Batesia y Panacea son muy similares y tienen como planta hospedera a Caryodendron spp. (DeVries et al., 1999; Hill et al., 2002; Daniels et al., 2008). A pesar que en la amazonia peruana Panacea prola es considerada como plaga en plantaciones de Caryodendron orinocense (Correa \& Vásquez, 2007; Gonzales \& Torres, 2010); esta mariposa posee potencial para los bionegocios, siendo comercializada como artesanía en países como Estados Unidos, con precios que superan los 25 dólares por cuadro. Actualmente existen algunos aspectos poco conocidos sobre la biología de esta especie que obstaculizan su crianza sostenible en cautiverio y semicautiverio. El presente estúdio contribuye al conocimiento de los aspectos biológicos de esta especie alimentada con hojas de Caryodendron orinocense Karts (Euphorbiaceae) en condiciones de cautiverio y semicautiverio para desarrollar crianzas masivas orientadas a la educación ambiental y los bionegocios (turismo y artesanías).

\section{MATERIAL Y MÉTODO}

El trabajo fue realizado desde Enero de 2007 hasta Enero de 2010, en la plantación experimental de "metohuayo", ubicado en el Centro de Investigaciones Allpahuayo, en el km 26.5 de la carretera Iquitos - Nauta, Iquitos-Perú (06758179561780 UTM). Las larvas de $P$. prola amazonica fueron colectadas manualmente en envases de plásticos cuando se alimentaban de las hojas de Caryodendron orinocense, conocida localmente como "metohuayo", luego se transportaron al laboratorio para ser confinados en envases apropiados hasta la obtención de los adultos para su identificación. Se efectuaron observaciones de comportamiento de vuelo, oviposición y número de huevos colocados. El ciclo biológico fue registrado en laboratorio a una temperatura de $27^{\circ} \mathrm{C}$, a partir de huevos recolectados en el campo. Las larvas emergidas se individualizaron en placas petri con papel humedecido y se las alimentó diariamente con trozos de hojas frescas de $C$. orinocense. La limpieza de las unidades de cría se realizó, inicialmente, cada dos días; durante los estadíos mayores, se efectuó diariamente, cambiando los discos de papel, y eliminando las excretas y el alimento sobrante, evitando así factores adversos de humedad y proliferación de entomopatógenos. A partir del V estadío fueron transferidas individualmente a envases de mayor tamaño para favorecer el empupamiento y un apropiado estiramiento alar en los adultos emergentes. Luego de obtenidos los adultos, se colocaron en un mariposario de $250 \mathrm{~m} 2$, que contenía plantas de $C$. orinocense y se les alimentó con miel y frutas variadas. El número de estadíos fue determinado a través de las mudas larvales y la medición del ancho de las cápsulas cefálicas. Los enemigos naturales como parasitoides fueron obtenidas a través de colectas de huevos y larvas de $P$. prola amazonica desde el campo y fueron confinadas en envases herméticos de plástico en condiciones de laboratorio y posteriormente se evaluó la emergencia de los parasitoides. Los depredadores de estados inmaduros y de adultos fueron colectados con la ayuda de redes entomológicas durante el proceso de depredación en condiciones de campo. El entomopatógeno fue obtenido durante la cría de larvas infectadas colectadas desde el campo y criadas en el laboratorio hasta la manifestación del patógeno. La determinación taxonómica de la mariposa fue realizada por Gerardo Lamas y los enemigos naturales uno fue determinado a nivel de genero por el especialista del grupo (G. Delvare) y los demás solo han sido determinados a nivel de familia.

\section{RESULTADOS Y DISCUSIÓN}

\section{COMPORTAMIENTO}

Los adultos de Panacea prola amazonica fueron observados volando en la plantación de $C$. orinocense, siendo muy activos entre las 10:00 y 13:30 h, efectuando vuelos ligeros y posándose en las hojas y el tronco de la planta hospedera. Se alimentan de frutos en descomposición; los machos, además, han sido observados succionando minerales del suelo húmedo, de excretas de animales y muy raras veces se alimentan del néctar de las flores. D'Abrera (1984) reporta para adultos del genero Panacea el consumo de carroña y excremento. La 
hembra deposita sus huevos en las hojas y en los tallos cerca de las hojas jóvenes. La oviposición está estrechamente relacionada a la presencia de brotes debido a que las larvas en los primeros estadios solo son capaces de alimentarse de hojas suaves.

\section{CICLO BIOLÓGICO.}

Los huevos son esféricos, con un diámetro promedio de 0,67 $\pm 0,05 \mathrm{~mm}$ y $0,71 \pm 0,04 \mathrm{~mm}$ de alto $(\mathrm{n}=20)$, de color amarillo intenso (Figura 2A), con 14 a 16 estrías bien pronunciadas distribuidas paralelamente; estos son colocados en una masa con un promedio de 262,80 $\pm 119.0(\mathrm{n}=10)$ huevos. Este estado dura tres días. La larva pasa por seis estadíos. En el primer estadío la larva es de color hialino, cabeza negra, sin cuernos, el cuerpo cubierto de pequeñas setas negras no urticantes, inmediatamente después de salir del huevo se dirigen hacia los brotes para alimentarse; tiene una duración promedio de $2,35 \pm 0,48$ días $(\mathrm{n}=20)$. En el segundo estadío el cuerpo comienza a tornarse de color anaranjado con abundantes manchas de color negro, en cada mancha se observa setas de color negro a manera de protuberancia, la cabeza es negra y se observan dos cuernos pequeños en la parte anterior de la cabeza. Este estadío dura en promedio $1,47 \pm 0,50$ días $(\mathrm{n}=$ 19). El tercer estadío dura en promedio $1,89 \pm 0,31$ días $(n=19)$ y es de color anaranjado, los cuernos de la cabeza se alargan y se caracteriza por una mancha blanca cerca al ápice, las setas que cubren el cuerpo inician su engrosamiento y son negras con una base ensanchada. El cuarto estadío dura en promedio 1,95 $\pm 0,22$ días $(\mathrm{n}=19)$ y es anaranjado, los cuernos de la cabeza y las setas del cuerpo crecen y se ensanchan, se tornan negras metálico, en la mayoría de las setas del cuerpo se observa 3 puntas con excepción de las setas dorsales que solo tiene una y los dos últimos segmentos poseen 5 puntas. El quinto estadío dura en promedio $3,11 \pm 0,31$ días $(\mathrm{n}=19)$ y es de color anaranjado, las setas del cuerpo crecen al igual que los cuernos de la cabeza. El ultimo estadío, con una duración promedio de 7,06 $\pm 1,43$ días $(\mathrm{n}=18)$, es de color anaranjado las setas del cuerpo crecen al igual que los cuernos de la cabeza; son más gruesas y fuertes, cada segmento tiene una banda de color azul metálico que cubre la parte dorsal y pleural de la larva (Figura 2B). La longitud máxima promedio alcanzada fue $51,9 \pm 3,8 \mathrm{~mm}(\mathrm{n}=18)$. Las medidas de las cápsulas cefálicas por cada estadío $(\mathrm{n}=10)$ se muestran en la Tabla 1. La prepupa pierde su color original tornándose amarillo pálido, se inmoviliza, acorta y se adhiere fuertemente desde la parte anal a un sustrato y se cuelga para desarrollar la pupa (Figura 2C). Dura en promedio 1,06 $\pm 0,23$ días ( $\mathrm{n}=$ 18). Este último estadio larval es crítico durante el proceso de cría, la prepupa es altamente sensible y el manipuleo ocasiona su muerte. La pupa es de color amarillo pálido o rosado pálido, con pintas negras distribuidas de forma longitudinal (Figura 2D), midiendo en promedio $32,5 \pm 1,5 \mathrm{~mm}(\mathrm{n}=16)$. Esta fase duró en promedio $8,73 \pm 0,45$ días $(\mathrm{n}=11)$. Los machos y las hembras presentan apariencias similares en la parte dorsal y ventral (Figura 2E y 2F). Los adultos nacen de 8.00 a $11.00 \mathrm{~h}$. los machos nacen primero y sobreviven 6 a 9 días y las hembras aproximadamente 30 días y la copula ocurre entre el tercer y séptimo día de la emergencia en cautiverio. La duración del ciclo, de huevo a adulto, fue 26-37 días en condiciones de laboratorio. Estos datos difieren de lo encontrado por Montoya (1989) que estudio el ciclo biológico de Panacea posible prola en Colombia con una duración de 38 a 55 días, con 8 estadios larvales. Aunque el huevo, larva y el adulto son muy similares puede tratarse de una subespecie diferente sin embargo Daniels et al., 2008 documentó 6 estadios larvales en Panacea procilla lysimache aunque no ha observado más estadios precisa que algunas larvas, excepcionalmente, pueden presentar más de 6 .

\section{ENEMIGOS NATURALES}

Se han encontrado 7 enemigos naturales de P. prola amazónica. i) El Hymenoptera, Scelionidae, que es una avispa que parasita los huevos de P. prola amazónica, es un insecto de color negro metálico de $0.2 \mathrm{~mm}$ de longitud, presenta dimorfismo sexual, la hembra posee antenas acodadas con abdomen ensanchado y los machos antenas filiformes con el abdomen angosto y corto. ii) Conura sp. Hymenoptera; Chalcididae es una avispa que parasita la pre pupa. Es de color amarillo con manchas negras en la parte dorsal del pterotorax, presenta un fémur ensanchado en la pata posterior, mide $6 \mathrm{~mm}$ de longitud. La pre-pupa parasitada no muere al instante; continúa su proceso hasta completar la fase de pupa. Los parásitos completan su desarrollo en el interior de la pupa y posteriormente emergen por un solo agujero, aproximadamente 30 avispas de una sola pupa. iii) El Hymenoptera; Vespidae es una avispa depredadora de larvas. De color marrón con el abdomen rojizo y mide $23 \mathrm{~mm}$ de longitud. Esta avispa aprovecha la inmovilidad de la larva durante el proceso de muda del cuarto al quinto estadio para morderla repetidamente hasta matarla luego lo atrapa y posteriormente vuela llevándose a su presa. iv) El Diptera, Tachinidae (Figura 3A) es una mosca endoparásito de larvas. Es de color negro, con abundantes pelos en el tórax y abdomen, las tibias presentan quetas. El abdomen es de color negro con bandas anchas perpendiculares de color blanco. Mide $8 \mathrm{~mm}$ de longitud. La mosca parasita larvas en 
estadios finales y después de completar su ciclo larval abandona la oruga muerta atravesando su piel para empupar en el suelo. v) El Diptera; Phoridae. Es una pequeña mosca de color negro mide aproximadamente $3 \mathrm{~mm}$ son endoparásitos de larvas. vi) El Diptera; Asilidae. Es una mosca grande que se alimenta de mariposas adultas. De color negro con manchas blancas en el tórax y en los fémures del primer par de patas, en el abdomen tiene 3 bandas blancas muy notorias, imita a una avispa de gran envergadura mide $25 \mathrm{~mm}$ de longitud, la mosca atrapa a su presa en vuelo la lleva a un sustrato y se alimenta de ella. El séptimo enemigo es un hongo entomopatógeno no determinado, que causa la muerte de la pupa. El micelio, de color blanco cubre la pupa de color blanco y a los pocos días el hongo desarrolla sus hifas en forma de prolongaciones muy pronunciadas que se extiende en todo el exterior de la pupa como espuelas.

\section{CONCLUSIONES}

La mariposa $P$. prola amazónica, posee un ciclo biológico relativamente corto y se puede criar con facilidad en condiciones de cautiverio (mariposario) y semicautiverio (plantación) por lo que posee un buen potencial para la exportación comercial, sin embargo en condiciones de semicautiverio se registró 7 enemigos naturales que merman su producción. Si colectamos los huevos y las larvas en estadios iniciales y los manejados en laboratorio se puede obtener una alta producción de pupas y mariposas sanas ya que sus enemigos naturales solo dañan los huevos y los últimos estadios larvales. En condiciones de cautiverio se evita la presencia de enemigos naturales y las condiciones apropiadas para su reproducción son: un espacio apropiado, la alimentación adecuada de los adultos y la presencia de brotes, de la planta hospedera, para estimular la oviposición. Se requieren aún estudios para determinar los índices de productividad.

\section{AGRADECIMIENTO}

Este trabajo fue financiado por INCAGRO a través del sub proyecto "Sistemas de crianza comunal de 6 especies de mariposas diurnas con alto potencial de exportación en la Región Loreto". Los autores agradecen al Dr G. Delvare (Cirad, Francia) quien identifico el Chalcididae, Milagros Rengifo, Patricia Ríos, Helmut Leibniz Rengifo Novoa, Edwin Miguel Risco Alzamora, Diana Bazán, Marelly Chuquival Navarro, Boney Espinar, Pedro Vela por el apoyo brindado durante el estudio, a Juan José Bellido Collahuacho por la revisión y comentarios y a Cesar Delgado por sus comentarios y aportes brindados que enriquecieron los resultados.

\section{BIBLIOGRAFÍA CITADA}

Correa R.; Vásquez J. 2007. El maravilloso mundo de las mariposas. Instituto de Investigaciones de la Amazonía Peruana. Iquitos, Perú. 49pp.

Checa, M.F.; Barragán, A.; Rodríguez, J.; Christman, M. 2009. Temporal abundance patterns of butterfly communities (Lepidoptera: Nymphalidae) in the Ecuadorian Amazonia and their relationship with climate. Annales de la Société Entomologique de France. 45(4): 470486.

D'Abrera, B. 1984. Butterflies of South America. Hill House. Melbourne, Australia. 255pp.

Daniels, J.C.; Rodriguez, E.; Whelan, J.C. 2008. The biology and immature stages of Panacea procilla lysimache (Lepidoptera: Nymphalidae) from Costa Rica, with the report of a new locality record. Tropical Lepidoptera Research 18(2): 70-73.

DeVries P. J.; Penz, C.M.; Walla, T.R. 1999. The biology of Batesia hypochlora from an Ecuadorian rainforest (Lepidoptera, Nymphalidae). Tropical Lepidoptera. 10(2): 43-46.

Fruhstorfer, H. 1915. Neue neotropische Nymphaliden. Societas Entomologica 30(12): 66.

Gonzáles, A.; Torres, G. 2010. Manual de cultivo de Metohuayo Caryodendron orinocense Karst. Instituto de Investigaciones de la Amazonía Peruana. Iquitos, Perú. 32pp.

Hill, R.I.; Penz, C.M; DeVries, P.J. 2002. Phylogenetic analysis and review of Panacea and Batesia butterflies (Nymphalidae). The Journal of the Lepidopterists' Society 56(4): 199-215.

Martinez, S.J.B. 1970. El inchi (Caryodendron orinocense Karst.) oleaginosa nativa de América Tropical. Universidad de Nariño. Departamento de Fitotecnia. Pasto, Colombia. 52 pp.

Montoya, D.C. 1989. Aspectos biológicos del gusano cachón del inchi (Panacea sp. pos. prola). Colombia Amazónica 4(1): 27-38.

Salazar, E.; Vargas J.I.; Mora, A.M.; Benavides, J. 2010. Identificación preliminar de los Rhophalocera que habitan en el Centro Experimental Amazónico (C.E.A.) Mocoa, Putumayo y algunas especies aptas para criar en cautiverio (Insecta: Lepidoptera). Bol.Cient.Mus.Hist.Nat. 14(1): 150-188.

Robbins, R.K.; Lamas, G.; Mielke, O.H.H.; Harvey, D.J.; Casagrande, M.M. 1996. Taxonomic composition and ecological structure of the species-rich butterfly community at Pakitza, Parque Nacional del Manu, Peru. In: Wilson, D.E.; Sandoval, A. (Eds). Manu: The biodiversity of Southeastern Peru. p. 217-252. 

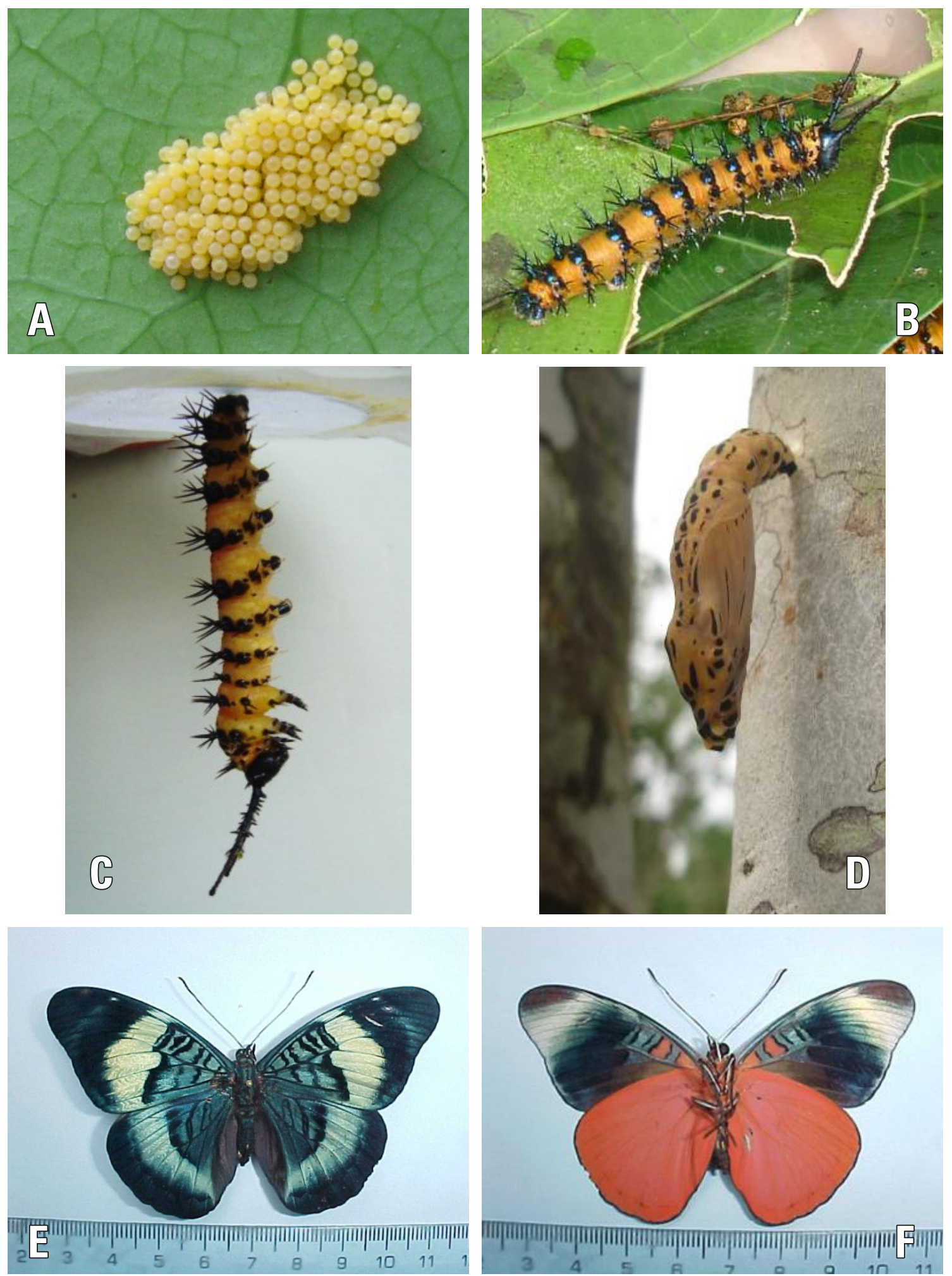

Figura 2. A Huevos de P. prola amazonica sobre hojas de Caryodendron orinocense; B. Larva de P. prola amazónica sobre hojas de Caryodendron orinocense; C. Pre pupa de P. prola amazónica; D. Pupa P. prola amazónica sobre tronco de Caryodendron orinocense; E Adulto vista dorsal P. prola amazonica; F. Adulto vista ventral P. prola amazónica. 

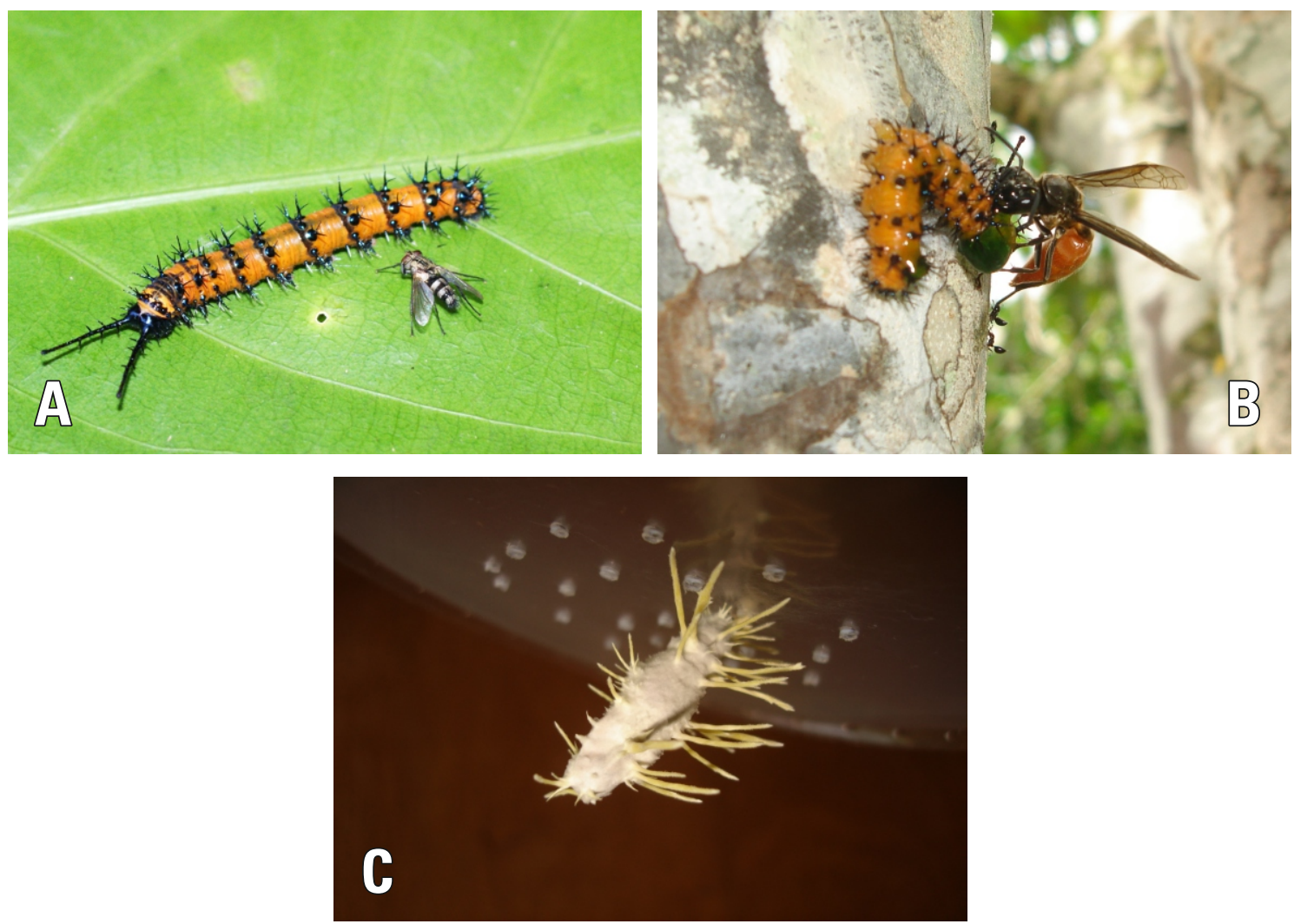

Figura 3. A. Mosca Tachinidae intentando parasitar una larva de $P$. prola amazonica en condiciones de plantación sobre hojas de Caryodendron orinocense; $\mathrm{B}$. avispa Hymenoptera atrapando una larva de $P$. prola amazónica desde la plantación de Caryodendron orinocense; C. Pupa muerta de $P$. prola amazónica por hongo entomopatógeno colectada de la plantación de Caryodendron orinocense.

Tabla 1. Medidas del ancho de la cápsula cefálica de larvas de Panacea prola amazonica $(\mathrm{n}=10)$.

\begin{tabular}{ccc}
\hline \multirow{2}{*}{ Estadío } & \multicolumn{2}{c}{ Ancho de la cápsula cefálica } \\
(mm)
\end{tabular}

